Ecología

\title{
Variación espacial y temporal de la infestación de la concha por Polydora sp. (Spionidae: Polychaeta) sobre la almeja mano de león (Nodipecten subnodosus) en la laguna Ojo de Liebre, Baja California Sur
}

Spatial and seasonal variation of shell infested by Polydora sp. (Spionidae: Polychaeta) on the lion's paw clam (Nodipecten subnodosus) in the lagoon Ojo de Liebre, Baja California Sur

\author{
Laura González-Ortiz $^{\mathrm{a}, *}$, Pablo Hernández-Alcántara ${ }^{\mathrm{b}}$, Ricardo Vázquez-Juárez ${ }^{\mathrm{c}}$, \\ Eduardo Quiroz-Guzmán ${ }^{c}$, Ma. Elena García-Garza ${ }^{a}$ y Jesús Ángel de León-González ${ }^{a}$ \\ ${ }^{a}$ Facultad de Ciencias Biológicas, Universidad Autónoma de Nuevo León, Apartado postal 5 «F», 66451, San Nicolás de los Garza, Nuevo León, México \\ ${ }^{\mathrm{b}}$ Instituto de Ciencias del Mar y Limnología, Universidad Nacional Autónoma de México, Circuito exterior s/n, Ciudad Universitaria, \\ 04510, Ciudad de México, México

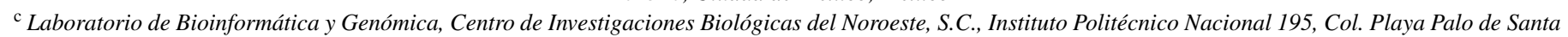 \\ Rita, 23090, La Paz, Baja California Sur, México \\ Recibido el 18 de marzo de 2016; aceptado el 23 de junio de 2017 \\ Disponible en Internet el 6 de diciembre de 2017
}

\begin{abstract}
Resumen
En la laguna Ojo de Liebre, Baja California Sur, México, se realizó un estudio sobre la infestación de Nodipecten subnodosus producida por Polydora sp., cuantificándose el daño y las variaciones del crecimiento de la almeja durante 3 años. Se realizaron 10 muestreos entre enero de 2013 y octubre de 2015, recolectándose 30 organismos por muestreo en los bancos Zacatoso, Chocolatero, El Dátil y La Concha. Se registraron entre 0 y 13 ampollas por concha, 1.77 ampollas concha ${ }^{-1}$ en promedio. El número de ampollas fue superior en las almejas provenientes de los bancos Chocolatero y Zacatoso, mientras que en los bancos El Dátil y La Concha no solamente disminuyó la infestación, sino que sus intervalos de variación fueron menores. Los bancos con las almejas más pequeñas fueron precisamente los que registraron el mayor número de ampollas, sobre todo el Chocolatero. A pesar de que el número máximo de ampollas se presentó en enero de 2013, mayo de 2014 y marzo de 2015 , la tendencia general fue de una reducción en la infestación de poliquetos en las almejas a lo largo del periodo de estudio.

(C) 2017 Universidad Nacional Autónoma de México, Instituto de Biología. Este es un artículo Open Access bajo la licencia CC BY-NC-ND (http://creativecommons.org/licenses/by-nc-nd/4.0/).
\end{abstract}

Palabras clave: Pectínidos; Bivalvos; Spionidae; Poliquetos perforadores; Ampollas de lodo

\section{Abstract}

In this study we evaluated the degree of infestation of Polydora sp. in Nodipecten subnodosus (lion's paw scallop). This research was carried out in laguna Ojo de Liebre, Baja California Sur, Mexico, quantifying the damage and growth of the clam for three years. A total of 10 samplings were carried out between January 2013 and October 2015, collecting 30 organisms by sampled at the banks Zacatoso, Chocolatero, El Dátil and La Concha. There were between 0 and 13 blisters per shell, 1.77 bisters shell ${ }^{-1}$ on average. The number of blisters was higher in the clams of the banks Chocolatero and Zacatoso, while in the banks El Dátil and La Concha, not only did the infestation decrease, but the variation intervals were

\footnotetext{
* Autor para correspondencia.

Correo electrónico: lauragortiz19@ hotmail.com (L. González-Ortiz).

La revisión por pares es responsabilidad de la Universidad Nacional Autónoma de México.
} 
smaller. Banks with smaller clams also recorded the largest number of blisters, mainly in Chocolatero. Although the maximum number of blisters occurred in January 2013, May 2014 and March 2015, the overall trend was for a reduction in polychaete infestation and clams over the length of study time.

(C) 2017 Universidad Nacional Autónoma de México, Instituto de Biología. This is an open access article under the CC BY-NC-ND license (http://creativecommons.org/licenses/by-nc-nd/4.0/).

Keywords: Pectinids; Bivalves; Spionidae; Burrow worms; Mud blisters

\section{Introducción}

Los moluscos tienen un alto valor comercial, ya que representan uno de los principales recursos pesqueros en el país; sin embargo, la sobreexplotación en algunas especies ha originado que en los bancos naturales disminuya considerablemente su número (Ramírez-Castillo, 2003). En el litoral del Pacífico mexicano se cuenta con más de 30 especies de pectínidos (Keen, 1971), pero de estos solo 3 son considerados de importancia comercial: la almeja catarina (Argopecten ventricosus), la almeja voladora (Pecten vogdesi) y la almeja mano de león (Nodipecten subnodosus) (Roldán-Carrillo, Maeda-Martínez, Massó-Rojas y Sicard-González, 2007). En particular, la presencia de esta última almeja ha venido decreciendo en los últimos años, y aunque no hay una causa aparente, se puede inferir que la sobreexplotación de la especie y su probable baja tasa de reproducción pueden ocasionar problemas en el desarrollo de su pesquería.

La distribución geográfica de la almeja mano de león comprende desde la laguna Manuela, Baja California, hasta Paita, Perú (Rombouts, 1991) y puede localizarse en lagunas, bahías y canales profundos con corrientes intensas (González-Estrada, 2003). Las poblaciones naturales de esta almeja en la laguna Ojo de Liebre, Baja California Sur, se han visto drásticamente afectadas hasta tal grado que se estableció una veda permanente desde 2012. Las causas de este fenómeno han sido atribuidas a varios factores como el cambio climático y su efecto en los factores ambientales ( $\mathrm{pH}$, ausencia de oxígeno, temperatura) y la infestación de invertebrados perforadores, entre otros.

La infestación por poliquetos espiónidos perforadores, particularmente los pertenecientes al complejo Polydora, constituye un serio problema para los moluscos tanto en el medio natural como en la acuicultura (Cremonte, 2011; Lauckner, 1983; Radashevsky, Lana y Nalesso, 2006). Cuando la intensidad de la infestación se incrementa frecuentemente se asocia con períodos de pobre desarrollo de los bivalvos (Silina y Zhukova, 2009), ya que el daño en la concha reduce su tasa de crecimiento (Silina, 2006), incrementando su mortalidad (Bergman, Elner y Risk, 1982). Existe un alto riesgo de infestación desde las etapas tempranas de su desarrollo, ya que los organismos perforadores inician su colonización con la fijación en la fase juvenil a las valvas de la almeja, y la construcción de tubos de lodo para refugiarse, posteriormente construyen madrigueras (Martin y Britayev, 1998). Al parecer, los sitios de mayor colonización están asociados con ambientes ricos en fitoplancton y materia orgánica, que proveen el alimento necesario para la presencia de los organismos perforadores.

El daño a la almeja mano de león se produce cuando el poliqueto perforador penetra en la superficie interna de la concha, y como respuesta a esta irritación el molusco tiende a secretar mayor cantidad de conquiolina con el fin de mantener aislado el lodo de la cavidad del manto. De esta manera se crean «ampollas de lodo», que le da mal aspecto a la concha y despiden un olor desagradable, por los metabolitos anaerobios de sulfato de hidrógeno, ocasionando una disminución de su valor comercial (Blake y Evans, 1973; Glasby et al., 2000; Handley y Bergquist, 1997; Martin y Britayev, 1998). Asimismo, el gasto energético durante la secreción de conquiolina retarda el crecimiento de la concha, aumentando su mortalidad (Almeida et al., 1996) y reduciendo su capacidad para acumular reservas nutricionales (Wargo y Ford, 1993).

La actividad perforadora de Polydora sobre las conchas de los moluscos puede ser realizada mediante 2 mecanismos: a) químico, por la secreción de fosfatasas ácidas y anhídridas carbónicas que disuelven o debilitan la matriz cálcica; y b) mecánico, utilizando las setas especializadas del quinto setígero. La combinación de ambos mecanismos permite la construcción de galerías en las cuales gradualmente se acumula lodo hasta alcanzar la cavidad del manto. Los bivalvos, como respuesta a esta afectación químico-mecánica, tienden a secretar mayor cantidad de conquiolina aislando el gusano de la cavidad del manto. Todo ello implica un fuerte gasto energético que debilita la condición fisiológica del bivalvo haciéndolo vulnerable a las enfermedades.

Los estudios que hasta el momento se han realizado para evaluar el nivel de infestación de organismos perforadores en moluscos bivalvos de importancia comercial son escasos. Se han realizado algunos trabajos generales sobre los poliquetos perforadores del género Polydora-Boccardia y su impacto en el cultivo de moluscos (Cáceres-Martínez, 2003), sobre las variaciones en los niveles de infestación y el desarrollo de las conchas (Ciocco, 1990; Ciocco et al., 2005; Silina, 2006). También se ha estudiado el porcentaje de infestación de Polydora en almejas de la Isla Margarita, Venezuela (Díaz-Díaz y Liñero-Arana, 2009) y el daño en conchas de Aequipecten tehuelche producido por la Polydora rickettsi en la Patagonia, Argentina (Diez, Orensanz, Márquez y Cremonte, 2013; Diez, Radashevsky, Orensanz y Cremonte, 2011).

En este sentido, el objetivo de este estudio fue evaluar el impacto de la presencia de poliquetos perforadores sobre la almeja mano de león en la laguna Ojo de Liebre, analizando sus variaciones espaciales y temporales. La presencia de los poliquetos en la almeja se cuantificó por medio del número de ampollas dentro de cada almeja, y el efecto sobre el molusco en poblaciones naturales fue evaluado por medio de los cambios observados en la longitud de la concha a lo largo de 3 años. 


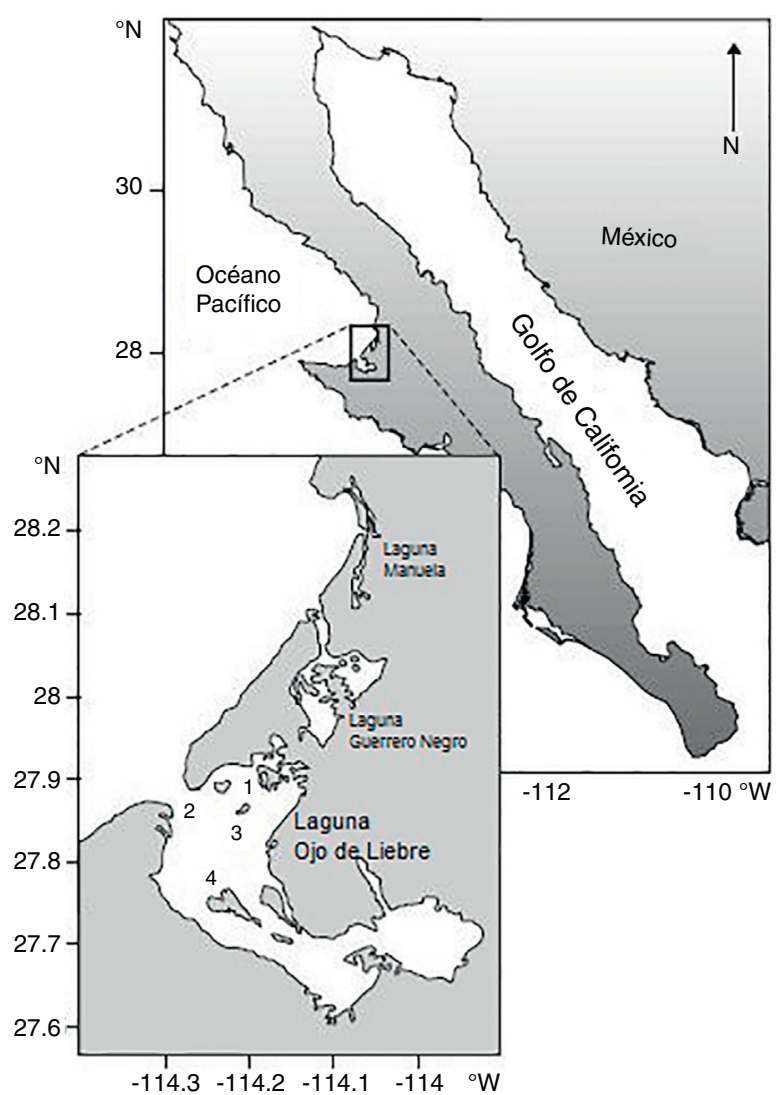

Figura 1. Ubicación de la laguna Ojo de Liebre, Baja California Sur y los bancos almejeros analizados: Zacatoso, Chocolatero, La Concha y El Dátil.

\section{Materiales y métodos}

Los muestreos se llevaron a cabo en la laguna Ojo de Liebre, Guerrero Negro, situada al Noroeste de Baja California Sur $\left(27^{\circ} 55^{\prime} \mathrm{N} ; 114^{\circ} 20^{\prime} \mathrm{O}\right)$ (fig. 1). Se recolectaron almejas en 4 bancos utilizados regularmente por los pescadores locales para la extracción de la almeja mano de león: Zacatoso, Chocolatero, El Dátil y La Concha. Se realizaron 10 muestreos entre enero de 2013 y octubre de 2015. En cada banco se capturaron en promedio 30 almejas vivas por muestreo, colocándolas en bolsas de polietileno selladas individualmente para ser procesadas posteriormente, aún con vida, en las instalaciones del Centro de Investigaciones Biológicas del Noroeste campus Guerrero Negro.

Con el fin de documentar el nivel de afectación se contabilizó el número de ampollas dentro de cada concha (figs. 2 A y B) y los poliquetos fueron extraídos fragmentando completamente las ampollas (figs. 2C y D); los organismos se cuantificaron, se etiquetaron y se fijaron en formol $10 \%$, finalmente se preservaron en alcohol al 70\%. La biometría de cada concha se realizó con un Vernier $(0.05 \mathrm{~mm}$ de precisión) midiendo su longitud total.

Las variaciones espaciales y temporales del número de ampollas por concha y la longitud de las almejas fueron analizadas con técnicas uni- y multivariadas usando el paquete estadístico Plymouth Routines in Multi-Variate Ecological Research (PRIMER v.7) (Clarke y Gorley, 2015). Se construyeron 2 matrices correspondientes al número de ampollas y a la longitud de almejas, cada una con 4 localidades y 10 meses de muestreo. Se realizaron análisis de varianza múltiple de 2 vías (Permanova) para probar las hipótesis de no diferencias en el número de ampollas y la no diferencia en la longitud de las almejas, en los

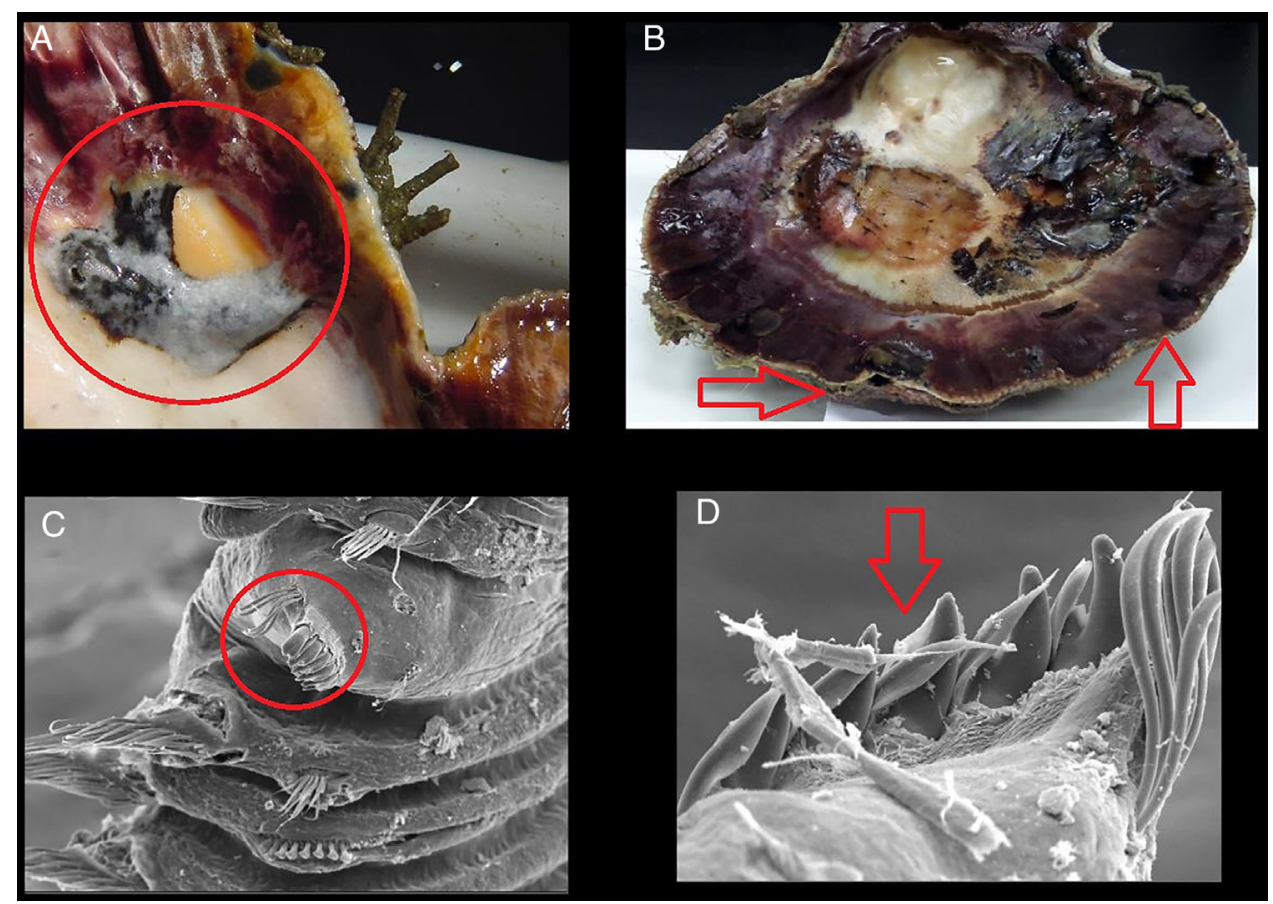

Figura 2. A. Presencia de ampollas de lodo en las valvas de la almeja mano de león. B. Crecimiento anormal de las valvas. C. Quinto setígero modificado del poliqueto Polydora sp. D. Detalle del quinto setígero modificado. 
diferentes bancos almejeros y a lo largo del periodo de muestreo ( 2 factores), con un nivel de significación $\alpha=0.05$. Esta técnica prueba las respuestas simultáneas de las variables a los factores designados en el diseño del análisis de varianza, de acuerdo con alguna medida de similitud y usando métodos permutacionales (Anderson, Gorley y Clarke, 2008). En la prueba del modelo completo se utilizó el índice de BrayCurtis para obtener las matrices de similitud y el diseño fue determinado por los factores fijos «banco» $\mathrm{y}$ «tiempo» (4 y 10 niveles, respectivamente) y su interacción. Las pruebas fueron realizadas con el paquete estadístico «Permanova + add» para «PRIMER v7» y los valores de Pseudo-F fueron obtenidos a partir de 999 permutaciones aleatorias. Para detectar las diferencias entre los bancos o entre los meses de muestreo se aplicaron comparaciones a posteriori «pair-wise» (Anderson et al., 2008). Estas comparaciones también se evaluaron por medio de la rutina Permanova, que realiza las pruebas tomando cada par de niveles a compararse, de uno en uno, y calcula una Pseudo-t como la raíz cuadrada de la Pseudo-F; los p-values para todas las comparaciones se obtuvieron por medio de 999 permutaciones aleatorias (Anderson et al., 2008).

Un aspecto relevante de la rutina Permanova es que no se realizan correcciones para las comparaciones múltiples. Es bien conocido que cuantas más comparaciones se realicen, mayor es la probabilidad de rechazar una o más hipótesis nulas simplemente por azar, sin embargo, los $P$-values obtenidos por permutaciones proveen directamente los valores de significación para contrastar cada hipótesis nula en forma individual. Según Anderson et al. (2008), aunque muchas correcciones podrían ser aplicadas en estos casos, como Bonferroni por ejemplo, no son necesariamente exactas y pueden ser excesivamente conservadoras, por lo que en este estudio se utilizaron directamente los valores de significación generados por las permutaciones.

\section{Resultados}

Durante el desarrollo de esta investigación se examinaron 1,011 almejas en 4 bancos almejeros, contabilizándose un total de 1,788 ampollas de lodo. Se registraron entre 0 y 13 ampollas por concha con un promedio de 1.77 ampollas concha ${ }^{-1}$ $\left(\mathrm{DE}=2.51\right.$ ampollas concha $\left.^{-1}\right)$.

El número de ampollas fue claramente superior en las almejas provenientes de los bancos Chocolatero (media $=3.03$ ampollas almeja $^{-1}, \quad \mathrm{DE}=2.67$ ampollas almeja $\left.^{-1}\right)$ y Zacatoso $\left(\right.$ media $=2.53$ ampollas almeja $^{-1}, \mathrm{DE}=3.08$ ampollas almeja $\left.^{-1}\right)$, sobre todo en el primero. Por el contrario, en los bancos El Dátil $\left(\right.$ media $=0.74$ ampollas almeja $^{-1}, \mathrm{DE}=1.53$ ampollas almeja $\left.^{-1}\right)$ y La Concha (media $=0.88$ ampollas almeja ${ }^{-1}, \mathrm{DE}=1.58$ ampollas almeja ${ }^{-1}$ ) la presencia de ampollas dentro de la almeja no solamente fue menor, sino que sus intervalos de variación también disminuyeron (fig. 3A).

A pesar de que las variaciones en tamaño de las conchas fueron diferentes en todos los bancos, se detectó que los bancos almejeros que registraron un número mayor de ampollas fueron precisamente los que presentaron las almejas más pequeñas (figs. 3 A y B). Es decir, las conchas recolectadas en los bancos Chocolatero $($ media $=7.79 \mathrm{~mm}, \mathrm{DE}=1.87 \mathrm{~mm}$ ) y Zacatoso
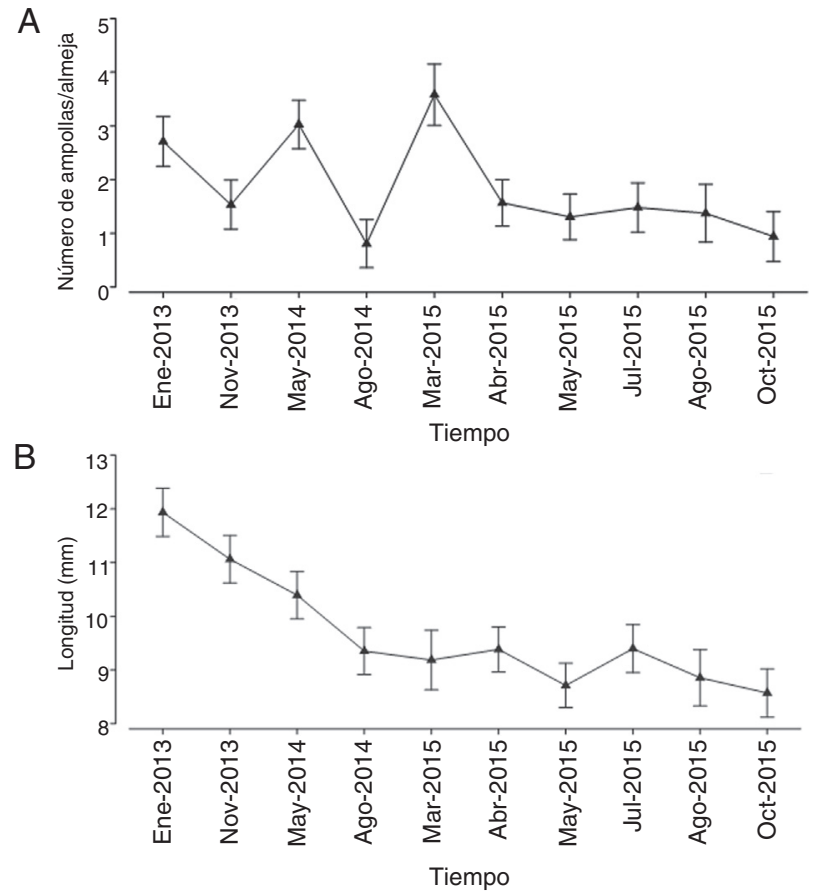

Figura 3. A. Valores promedio del número de ampollas por almeja y su variabilidad en cada uno de los bancos almejeros. B. Valores promedio de la longitud de las almejas y su variabilidad en cada uno de los bancos almejeros.

(media $=9.35 \mathrm{~mm}, \mathrm{DE}=2.32 \mathrm{~mm}$ ) tuvieron una longitud menor, sobre todo en el primero, que las conchas provenientes de los bancos El Dátil (media = $11.46 \mathrm{~mm}, \mathrm{DE}=2.26 \mathrm{~mm}$ ) y La Concha (media $=10.13 \mathrm{~mm}, \mathrm{DE}=2.24 \mathrm{~mm}$ ) que registraron los mayores tamaños (fig. 3B).

A lo largo del tiempo la presencia de ampollas fue muy irregular, aunque a partir de abril de 2005 se registró una tendencia constante en la reducción del número de ampollas por almeja (fig. 3A). A pesar de observarse un mayor número de ampollas en enero de 2013 (media $=2.71 \mathrm{~mm}$ ), mayo de 2014 $($ media $=3.03 \mathrm{~mm})$ y marzo de $2015($ media $=3.58 \mathrm{~mm})$, la tendencia general fue de una reducción en el nivel de infestación por poliquetos a lo largo del tiempo.

Por otra parte, las variaciones en la longitud de las conchas mostraron una reducción constante en su tamaño promedio a lo largo del periodo de muestreo, disminuyendo desde $11.94 \mathrm{~mm}$ en enero de 2013 hasta $8.57 \mathrm{~mm}$ en octubre de 2015 (fig. 3B). Al parecer, con el paso del tiempo el tamaño de las conchas de la almeja mano de león han tendido a ser más pequeñas.

Las variaciones espaciales y temporales en la presencia de poliquetos dentro de la almeja, evaluadas por medio del análisis múltiple de varianza de 2 vías (Permanova), mostraron que, en efecto, sí hubo diferencias significativas entre los bancos almejeros (Pseudo- $F=79.40, p=0.001$ ) (tabla 1). Los bancos Chocolatero y Zacatoso presentaron claramente un mayor número de ampollas, y a pesar de que los 2 registraron valores promedio similares (3.03 y 2.53 ampollas almeja ${ }^{-1}$, respectivamente), sus diferencias fueron suficientes para ser considerados como grupos distintos (Pseudo- $t=2.50, p=0.016$ ). Las diferencias registradas entre los bancos Chocolatero y Zacatoso determinaron que prácticamente todas las comparaciones entre 
Tabla 1

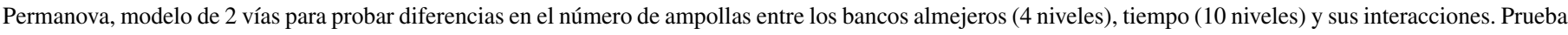
a posteriori «pair-wise» entre bancos almejeros y entre meses de muestreo.

\begin{tabular}{|c|c|c|c|c|c|c|}
\hline Fuente & df & SS & MS & Pseudo-F & p (permutación) & Permutaciones únicas \\
\hline Banco & 3 & 874.70 & 291.57 & 79.40 & 0.001 & 999 \\
\hline Tiempo & 9 & 643.54 & 71.84 & 19.56 & 0.001 & 999 \\
\hline Banco $\times$ tiempo & 27 & 1084.30 & 40.16 & 10.94 & 0.001 & 998 \\
\hline Residuales & 971 & 3565.60 & 3.67 & & & \\
\hline Total & 1,010 & 6387.8 & & & & \\
\hline
\end{tabular}

Prueba por pares entre bancos almejeros

\begin{tabular}{|c|c|c|}
\hline Grupos & Pseudo-t & $\mathrm{p}$ (permutación) \\
\hline Zacatoso, Chocolatero & 2.50 & 0.016 \\
\hline Zacatoso, El Dátil & 9.81 & 0.001 \\
\hline Zacatoso, La Concha & 9.85 & 0.001 \\
\hline Chocolatero, El Dátil & 11.46 & 0.001 \\
\hline Chocolatero, La Concha & 1.74 & 0.001 \\
\hline El Dátil, La Concha & 0.96 & 0.334 \\
\hline
\end{tabular}

Prueba por pares entre meses de muestreo

\begin{tabular}{|c|c|c|}
\hline Grupos & Pseudo-t & p (permutación) \\
\hline Ene-2013, Nov-2013 & 56.19 & 0.001 \\
\hline Nov-2013, May-2014 & 44.61 & 0.001 \\
\hline May-2014, Ago-2014 & 67.58 & 0.001 \\
\hline Ago-2014, Mar-2015 & 77.52 & 0.001 \\
\hline Mar-2015, Abr-2015 & 49.02 & 0.001 \\
\hline Abr-2015, May-2015 & 13.95 & 0.180 \\
\hline May-2015, Jul-2015 & 0.24 & 0.809 \\
\hline Jul-2015, Ago-2015 & 0.44 & 0.680 \\
\hline Ago-2015, Oct-2015 & 10.07 & 0.299 \\
\hline
\end{tabular}

Los valores negrita indican diferencias significativas.

los bancos almejeros fueran significativamente distintas, con excepción de El Dátil y La Concha (tabla 1). En estos 2 últimos bancos claramente se registró un menor número de ampollas (0.74 y 0.88 ampollas almeja ${ }^{-1}$ en promedio, respectivamente), sin embargo, sus diferencias no fueron estadísticamente significativas y no pudieron ser discriminados entre sí (Pseudo- $t=0.96$, $p=0.334)$.

El análisis Permanova confirmó la existencia de diferencias significativas en el número de ampollas a lo largo del tiempo (Pseudo-F $=19.56, p=0.001$ ) (tabla 1 ). La amplia variación observada en el número de ampollas en los primeros meses del periodo de muestreo, entre enero de 2013 y marzo de 2015 (fig. 3A) definió en gran medida la diferencia significativa generada por el modelo multivariado completo. El número de ampollas en las almejas se redujo con el paso del tiempo (fig. 3A) y en efecto, las pruebas «pair-wise» a partir de abril de 2015 confirmaron que en los últimos meses de estudio las diferencias en el número de ampollas entre cada mes de muestreo no fueron significativas (tabla 1).

El patrón de variación en el tamaño de las conchas entre los bancos almejeros fue distinto del registrado por los cambios espaciales del número de ampollas, ya que si bien el modelo de varianza múltiple también detectó diferencias significativas en la longitud de las almejas entre los bancos (Pseudo- $F=167.43$, $p=0.001$ ) (tabla 2), en este caso las longitudes de las conchas fueron distintas entre cada uno de los bancos (tabla 2). Es decir, a pesar de que en Chocolatero y Zacatoso las conchas fueron más pequeñas, y en El Dátil y La Concha fueron más grandes, en cada uno de los bancos se presentaron poblaciones de almejas con tamaños significativamente distintos (tabla 2).

Las pruebas «pair-wise» realizadas para detectar las diferencias en la longitud de las almejas durante los meses de muestreo mostraron que la mayoría de los cambios en el tamaño de las conchas a lo largo del tiempo también fueron significativamente diferentes (tabla 2). Al inicio de periodo de estudio (enero de 2013 a mayo de 2014) las conchas fueron significativamente más grandes, pero su longitud descendió gradualmente con el paso del tiempo (fig. 3B). A partir de agosto de 2014 las conchas fueron más pequeñas que en los primeros meses del muestreo y su longitud se mantuvo con poca variación hasta abril de 2015, periodo durante el cual la prueba «pair-wise» no detectó diferencias significativas (tabla 2). A partir de abril de 2015 y hasta el fin del estudio (octubre de 2015) las conchas mantuvieron su tendencia a ser más pequeñas, pero en este caso las diferencias sí fueron estadísticamente significativas. Este patrón de variación refleja cambios diferenciales en el tamaño de las conchas a lo largo del tiempo, pero también muestra que el crecimiento de las almejas ha ido disminuyendo sistemáticamente.

\section{Discusión}

En términos generales, el porcentaje de infestación en el banco Chocolatero fue del $40 \%$, que precisamente fue el banco donde se registraron las menores tallas $(4.5-8 \mathrm{~cm})$ durante la 
Tabla 2

Permanova, modelo de 2 vías para probar diferencias en la longitud de las almejas entre los bancos almejeros (4 niveles), tiempo (10 niveles), y sus interacciones. Prueba a posteriori «pair-wise» entre bancos almejeros y entre meses de muestreo.

\begin{tabular}{lllllll}
\hline Fuente & df & SS & MS & Pseudo-F & p (permutación) & Permutaciones únicas \\
\hline Banco & 3 & 1600.70 & 533.56 & 167.43 & $\mathbf{0 . 0 0 1}$ & 998 \\
Tiempo & 9 & 966.16 & 107.35 & 33.69 & $\mathbf{0 . 0 0 1}$ & 998 \\
Banco x Tiempo & 27 & 720.72 & 26.69 & 8.38 & $\mathbf{0 . 0 0 1}$ & 997 \\
Residuales & 971 & 6483.40 & 3.19 & & & \\
Total & 1,010 & 6387.80 & & & &
\end{tabular}

Prueba por pares entre bancos almejeros

\begin{tabular}{lll}
\hline Grupos & Pseudo-t & p (permutación) \\
Zacatoso, Chocolatero & $\mathbf{1 0 . 0 2}$ & $\mathbf{0 . 0 0 1}$ \\
Zacatoso, El Dátil & $\mathbf{1 3 . 2 3}$ & $\mathbf{0 . 0 0 1}$ \\
Zacatoso, La Concha & $\mathbf{5 . 3 3}$ & $\mathbf{0 . 0 0 1}$ \\
Chocolatero, El Dátil & $\mathbf{2 2 . 5 9}$ & $\mathbf{0 . 0 0 1}$ \\
Chocolatero, La Concha & $\mathbf{1 4 . 9 3}$ & $\mathbf{0 . 0 0 1}$ \\
El Dátil, La Concha & $\mathbf{8 . 0 3}$ & $\mathbf{0 . 0 0 1}$
\end{tabular}

Prueba por pares entre meses de muestreo

\begin{tabular}{lll}
\hline Grupos & Pseudo-t & p (permutación) \\
Ene-2013, Nov-2013 & $\mathbf{5 . 0 3}$ & $\mathbf{0 . 0 0 1}$ \\
Nov-2013, May-2014 & $\mathbf{3 4 . 1 6}$ & $\mathbf{0 . 0 0 1}$ \\
May-2014, Ago-2014 & $\mathbf{2 7 . 1 1}$ & $\mathbf{0 . 0 0 7}$ \\
Ago-2014, Mar-2015 & 0.45 & 0.663 \\
Mar-2015, Abr-2015 & 0.10 & 0.924 \\
Abr-2015, May-2015 & $\mathbf{2 6 . 0 7}$ & $\mathbf{0 . 0 0 9}$ \\
May-2015, Jul-2015 & $\mathbf{4 6 . 0 6}$ & $\mathbf{0 . 0 0 1}$ \\
Jul-2015, Ago-2015 & $\mathbf{3 4 . 6 8}$ & $\mathbf{0 . 0 0 1}$ \\
Ago-2015, Oct-2015 & $\mathbf{1 9 . 7 0}$ & $\mathbf{0 . 0 4 9}$ \\
\hline
\end{tabular}

Los valores en negrita indican diferencias significativas.

mayoría de los meses de muestreo. Estos valores de infestación fueron similares a los observados en otros estudios realizados en almejas de diferentes especies. Algunos estudios que evaluaron el porcentaje de afectación de los poliquetos perforadores sobre diferentes especies de bivalvos en maricultivos detectaron daños de alrededor del 50\% en Crassostrea virginica de la bahía Delaware (Wargo y Ford, 1993), mientras en C. gigas se observó que hasta el $57 \%$ de las conchas se encontraron infestadas (Handley, 1995). Por el contrario, los valores registrados en la laguna Ojo de Liebre fueron inferiores a los observados en otros estudios: en Nueva Zelanda se detectó que hasta el 69.4\% de las conchas estaban infestadas (Handley y Bergquist, 1997); estudios realizados en 6 bivalvos de importancia comercial en el norte de la Patagonia, Argentina (Aequipecten tehuelchus, Mytilus sp., Aulacomya atra, Ostrea puelchana, Prothothaca antigua y Pododesmus rudis) registraron que el daño producido por Polydora rickettsi fue del 54\% (Diez et al., 2011); investigaciones realizadas con la escalopa tehuelche (Chlamys tehuelcha) registraron un $46 \%$ de infestación producida, en este caso, por Polydora websteri en el golfo de San José (Chubut), Argentina (Ciocco, 1990), y en el caso del ostión Crassostrea gigas se detectó un nivel de afectación del $60 \%$, producida por $P$. websteri en la laguna Barra de Navidad, Jalisco (Gallo-García, García-Ulloa, Rejón-Aviña, Godínez-Sordia y Díaz, 2007). Los estudios llevados a cabo en bivalvos en ambientes naturales también registraron altos niveles de infestación, ya que en la laguna Restinga en Venezuela se observaron valores de hasta el 64.57\% en conchas de Crassostrea rhizophorae debido a la presencia de P. websteri (Díaz-Díaz y Liñero-Arana, 2009).

La presencia de un mayor número de ampollas en conchas de menor talla (Ciocco, 1990), como lo observado a lo largo de la presente investigación en el banco Chocolatero, puede ser resultado de que estas reducen el índice de condición nutricional en el bivalvo al disminuir su habilidad de acumular reservas nutricionales a lo largo del tiempo (Wargo y Ford, 1993). Naturalmente, el efecto de los poliquetos sobre el hospedero depende de la intensidad de la infestación y del nivel del daño causado a la concha, pero en términos generales, la presencia de los poliquetos retarda el desarrollo de la almeja y reduce el tamaño de la gónada (Handley y Bergquist, 1997; Simon, Ludford y Wynne, 2006). Precisamente, en el banco Chocolatero de la laguna Ojo de Liebre las tallas que prevalecieron durante la mayoría de los meses de muestreo fueron las más pequeñas de la laguna, presentando, además, visibles malformaciones en las valvas (figs. $2 \mathrm{~A}$ y B). Asimismo, el gasto energético del molusco se orienta principalmente a la secreción de conquiolina alrededor de las ampollas de lodo, ocasionando afectaciones directas en su crecimiento y reproducción, y finalmente, un aumento en sus tasas de mortalidad (Almeida et al., 1996).

Los niveles de almacenamiento y utilización de energía están fuertemente ligados a la disponibilidad de alimento, pero también a los ciclos reproductivos de los bivalvos (Gabbott, 1983; Sastry, 1979). La gametogénesis es un proceso que demanda mucha energía, que puede ser obtenida directamente del 
alimento (sestón) o de los órganos de almacenamiento, como la glándula digestiva (que almacena principalmente lípidos) y el músculo abductor (que almacena principalmente hidratos de carbono en forma de glucógeno y proteína) (Ansell, 1974; Barber y Blake, 1983; Gabbott, 1975).

En general, los tamaños pequeños de las almejas prevalecieron en los meses de marzo a mayo, probablemente debido a que los procesos de gametogénesis durante este periodo dependen básicamente de las reservas almacenadas en el músculo abductor y de su calidad nutricional para formar depósitos de glucógeno (Arellano-Martínez, 2005). Esto tiene un efecto directo sobre los niveles de infestación, ya que al tener un mayor gasto de energía y una baja calidad nutricional, las almejas son más susceptibles a la presencia de organismos perforadores oportunistas. Hay que recordar que la almeja mano de león llega a su madurez sexual, en un $90 \%$ de los organismos, en el mes agosto (Arellano-Martínez, 2005).

El mayor porcentaje de infestación, alcanzando hasta el $40 \%$ de las almejas, se registró en los bancos Chocolatero y Zacatoso, encontrándose una relación directa entre el tamaño de la concha y el número de ampollas. Sin embargo, para determinar completamente las causas que originan este tipo de relaciones es necesario llevar a cabo estudios detallados sobre el efecto de las variaciones ambientales sobre la presencia y distribución de las especies perforadoras en el área de estudio, pero también determinar si los cambios climáticos influyen sobre las tasas de infestación de las almejas mano de león.

En conclusión, se puede decir que la mayor infestación de poliquetos en las almejas de menor tamaño ocurrió en el banco Chocolatero, básicamente en los meses de enero de 2015 y entre los meses de mayo de2014 a abril de 2015, debido a que en este período la almeja mano de león inicia su actividad reproductiva y de desove, reduciendo su condición nutricional y estando más expuesta a la presencia de organismos perforadores, y a que este banco se localiza en la boca de la laguna, en donde muy probablemente la influencia del flujo de las corrientes marinas no permitieron que se desarrollaran como en otros bancos almejeros.

\section{Agradecimientos}

Agradecemos el apoyo brindado, al director $\mathrm{M}$. en $\mathrm{C}$. Everardo Mariano Meléndez y al personal del Área Natural Protegida Reserva de la Biosfera del Vizcaíno por facilitar el acceso a la laguna Ojo de Liebre, Baja California Sur, México, y a la cooperativa pesquera por su apoyo logístico durante los muestreos realizados. Agradecemos al Centro de Investigaciones Biológicas del Noroeste, Unidad Guerrero Negro, por facilitarnos el laboratorio para procesar las muestras, en especial al Dr. Rogelio Ramírez Serrano y a la Bióloga Marina Gabriela Valle Meza.

\section{Referencias}

Almeida, M. J., Moura, C., Machado, J., Coimbra, J., Vilarinho, L., Ribeiro, C., et al. (1996). Aminoacid and metal content of Crassostrea gigas shell infested by Polydora sp. in the prismatic layer insoluble matrix and blister membrane. Aquatic Living Resources, 9, 179-186.
Anderson, M. J., Gorley, R. N. y Clarke, K. R. (2008). Permanova + for PRIMER: guide to software and statistical methods. Plymouth, UK: PRIMER-E.

Ansell, A. (1974). Seasonal changes in biochemical composition of the bivalve Chlamys septemradiata from the Clyde Sea area. Marine Biology, 25, 85-99.

Arellano-Martínez, M. (2005). Características ecofisiológicas de la reproducción de Nodipecten subnodosus (Pectinidae) en la laguna Ojo de Liebre, B.C.S., México (Tesis doctoral). Centro Interdisciplinario de Ciencias Marinas. Instituto Politécnico Nacional. La Paz, Baja California Sur, México.

Barber, B. J. y Blake, N. J. (1983). Growth and reproduction of the bay scallop, Argopecten irradians (Lamarck) at its southern distributional limit. Journal of Experimental Marine Biology and Ecology, 66, 247-256.

Bergman, K. M., Elner, R. W. y Risk, M. J. (1982). The influence of Polydora websteri borings on the strength of the shell of the sea scallop Placopecten magellanicus. Canadian Journal of Zoology, 60, 2551-2556.

Blake, J. A. y Evans, J. W. (1973). Polydora and related genera as borers in mollusc shells and other calcareus substrates. Veliger, 15, 235-249.

Cáceres-Martínez, J. (2003). Gusanos perforadores de los géneros Polydora y Boccardia y su impacto en el cultivo de moluscos. Boletín del Programa Nacional de Sanidad Acuícola y la Red de Diagnóstico, 4, 1-6.

Ciocco, N. F. (1990). Infestación de la Vieyra tehuelche (Chlamys tehuelcha) por Polydora websteri Hartman (Polychaeta: Spionidae) en el Golfo San José (Chubut, Argentina): un enfoque cuantitativo. Biología Pesquera, 19, 9-18.

Ciocco, N. F., Lasta, M. L., Narvarte, M., Bremec, C., Bogazzi, E. y Valero, J. (2005). The tehuelche scallop Aequipecten tehuelchus. En S. E. Shumway y G. J. Parsons (Eds.), Scallops: biology, ecology and aquaculture (2nd ed, pp. 1251-1292). Argentina: Elsevier

Clarke, K. R. y Gorley, R. N. (2015). Primer version 6: user manual/tutorial. Plymouth: Primer-E Ltd.

Cremonte, F. (2011). Enfermedades de moluscos bivalvos de interés comercial causadas por metazoos. En Enfermedades de moluscos bivalvos de interés en Acuicultura (pp. 331-385), Barcelona: Fundación Observatorio Español de Acuicultura.

Díaz-Díaz, O. y Liñero-Arana, I. (2009). Percentage of infestation level of Polydora cf. websteri Hartman, 1943 (Polychaeta: Spionidae) by size class of Crassostrea rhizhophorae (Guilding, 1828) on La Restinga Lagoon (Margarita Island, Venezuela). Revista Científica, FCV-LUZ, 19, 113-118.

Diez, M. E., Orensanz, J. M., Márquez, F. y Cremonte, F. (2013). Shell damage in the Tehuelche scallop Aequipecten tehuelchus caused by Polydora rickettsi (Polychaeta: Spionidae) infestation. Journal of Invertebrate Pathology, 114, 107-113.

Diez, M., Radashevsky, E. V. I., Orensanz, J. M. y Cremonte, F. (2011). Spionid polychaetes (Annelida: Spionidae) boring into shells of molluses of commercial interest in northern Patagonia, Argentina. Italian Journal of Zoology, 78, 497-504.

Gabbott, P. A. (1975). Storage cycles in marine bivalve molluscs: a hypothesis concerning the relationship between glycogen metabolism and gametogenesis. En H. Barnes (Ed.), Nineth European Marine Biology Symposium (pp. 191-211). Aberdeen University Press.

Gabbott, P. A. (1983). Development and seasonal metabolic activities in marine molluscs. En P. W. Hochachka (Ed.), The Mollusca, 2 (pp. 165-217). Londres: Academic Press.

Gallo-García, M. C., García-Ulloa, M., Rejón-Aviña, A., Godínez-Sordia, D. E. y Díaz, A. H. (2007). Infestación de espiónidos perforadores en el ostión Crassostrea gigas cultivado en la laguna de Barra de Navidad, Jalisco, México. Avances en Investigación Agropecuaria, 11, 63-73.

Glasby, C. J., Fauchald, P. A., Paxton, K., Rouse, H., Rousell, G. y Wilson, R. (2000). Class Polychaeta. En P. L. Beesley, G. Ross, y C. J. Glasby (Eds.), Polychaetes and allies: the southern synthesis. Fauna of Australia IV A. Polychaeta, Myzostimida, Pogonophora, Echiura, Sipuncula (pp. 1-296). Melbourne: CSIRO Publishers.

González-Estrada, F. (2003). Temperaturas letales y temperatura óptima para el crecimiento en una población de almeja mano de león (Nodipecten subnodosus Sowerby, 1883) (Tesis de maestría). Centro de investigaciones Biológicas del Noroeste S.C., La Paz, B. C. S., México.

Handley, S. J. (1995). Spionid polychaetes in Pacific oysters, Crassostrea gigas (Thunberg) from Admiralty Bay, Marlborough Sounds, New Zealand. New Zealand Journal Marine Freshwater Research, 29, 305-309. 
Handley, D. J. y Bergquist, P. R. (1997). Spionid Polychaete infestation of intertidal Pacific oysters Crassostrea gigas (Thunberg), Mahurangi Harbour, northern New Zealand. Aquaculture, 153, 191-205.

Keen, A. M. (1971). Sea shells of tropical West America. Stanford: Stanford University Press.

Lauckner, G. (1983). Diseases of Mollusca: Bivalvia. En O. Kinne (Ed.), Diseases of marine animals (pp. 477-879). Hamburg: Biologische Anstalt Helgoland.

Martin, D. T. A. y Britayev, T. A. (1998). Symbiotic polychaetes review of known species. En A. D. Ansell, R. N. Gibson y M. Barnes (Eds.), Oceanography and marine biology: annual review, 36, 217-340.

Radashevsky, V. I., Lana, P. C. y Nalesso, R. C. (2006). Morphology and biology of Polydora species (Polychaeta: Spionidae) boring into oyster shells in South America, with the description of a new species. Zootaxa, 1353, $1-37$.

Ramírez-Castillo, E. R. (2003). Desarrollo reproductivo estacional de la almeja mano de león Nodipecten subnodosus (Tesis). Instituto Tecnológico Agropecuario Núm. 21 Bacum, Sonora., México.

Roldán-Carrillo, L. M., Maeda-Martínez, A. N., Massó-Rojas, A. y SicardGonzález, M. T. (2007). Salinity tolerance and resistance of the Pacific lion's paw scallop Nodipecten subnodosus and the relationships with species distribution and density in a coastal lagoon. Journal of Shellfish Research, 24 353-362.

Rombouts, A. (1991). Guide to pectin shells. Recent Pectinadae and Propeamussidae of the world. Oegstgeest, Países Bajos: Universal Book Services, Dr. W. Backuys.

Sastry, A. N. (1979). Pelecypoda (excluding Ostreidae). En A. C. Giese y J. S. Pearse (Eds.), Reproduction of marine invertebrates (pp. 113-292). New York: Academic Press.

Silina, A. V. (2006). Tumor-like formations on the shells of Japanese scallops Patinopecten yessoensis (Jay). Marine Biology, 148, 833-840.

Silina, A. V. y Zhukova, N. V. (2009). Topical and trophic relationships in a boring polychaete-scallop association: fatty acid biomarker approach Marine Ecology Progress Series, 394, 125-136.

Simon, C. A., Ludford, A. y Wynne, S. (2006). Spionid polychaetes infesting cultures abalone, Haliotis midae, in South Africa. African Journal of Marine Science, 28, 167-171.

Wargo, R. N. y Ford, S. E. (1993). The effect of shell infestation by Polydora sp. and infection by Haplosporidium nelsoni (MSX) on the tissue condition oysters, Crassostea virginica. Estuaries, 16, 229-234. 